Supporting information

\title{
Lysozyme adsorption on porous organic cages: a molecular simulation study
}

Daohui Zhao ${ }^{1,2^{*}}$, Yuqing Wang ${ }^{1}$, Qianwen Su${ }^{1,2}$, Libo Li' ${ }^{2}$, Jian Zhou ${ }^{2 *}$

${ }^{1}$ Hubei Collaborative Innovation Center for Advanced Organic Chemical Materials,

Ministry of Education Key Laboratory for the Synthesis and Application of Organic

Functional Molecules, School of Chemistry and Chemical Engineering, Hubei

University, Wuhan 430062, P.R. China

${ }^{2}$ School of Chemistry and Chemical Engineering, Guangdong Provincial Key Lab for Green Chemical Product Technology, South China University of Technology,

Guangzhou 510640, P. R. China

*Corresponding author:

Jian Zhou

Tel.: (+86) 2087114069

E-mail: jianzhou@scut.edu.cn

Daohui Zhao

Tel.: (+86) 18827412435

E-mail: zdh@hubu.edu.cn 


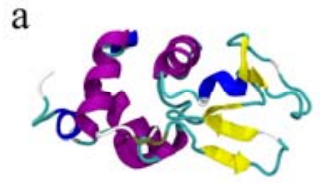

$\mathrm{O} 1$

d

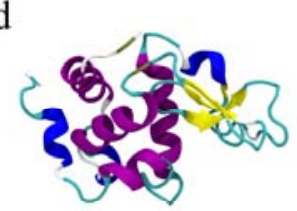

O4 b

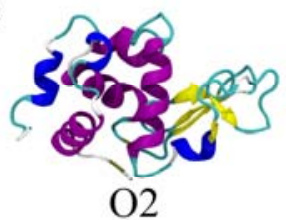

$\mathrm{e}$

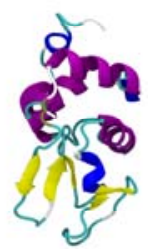

$\mathrm{O} 5$ $\mathrm{c}$

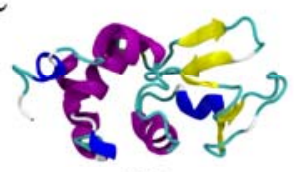

O3

f

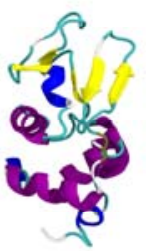

O6

Figure S1. Six different initial orientations of lysozyme adsorption on the CC3 surface.
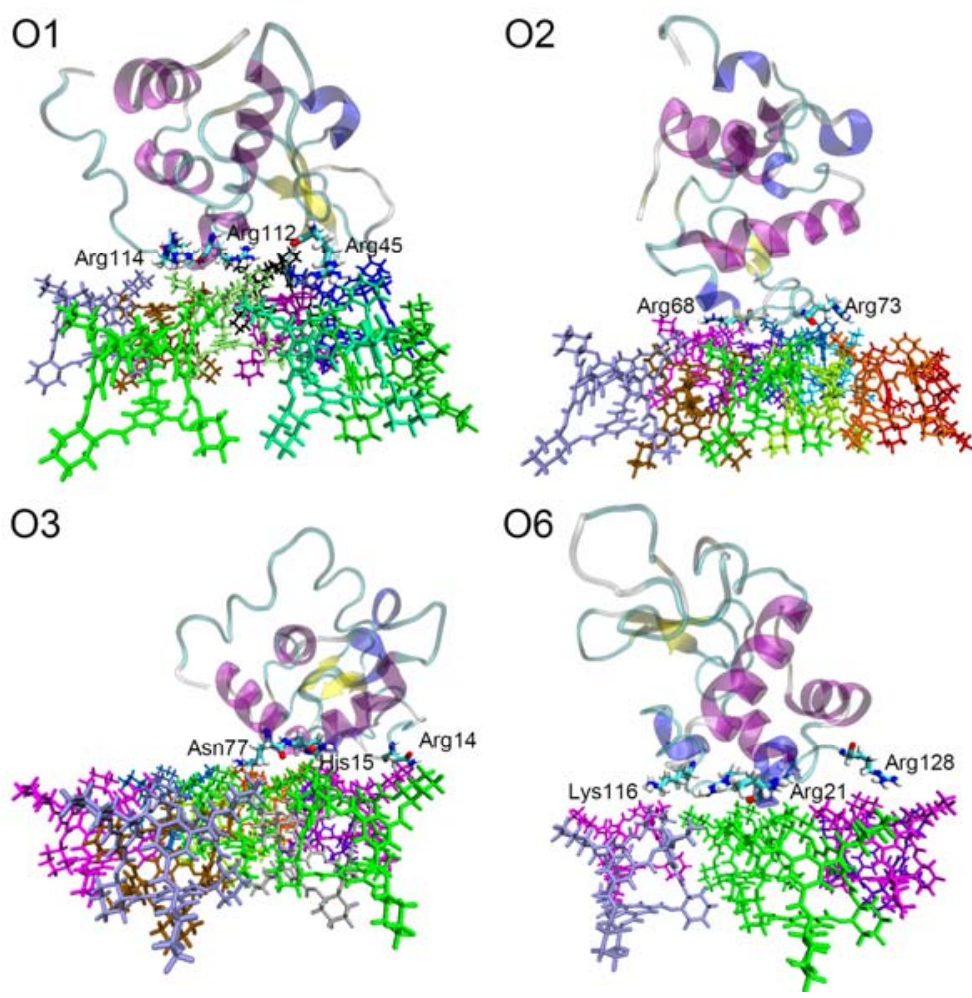

Figure S2. Illustrations for arginine amino acids that mediate the lysozyme adsorption on CC3 surface for O1, O2, O3, and O6 systems. 

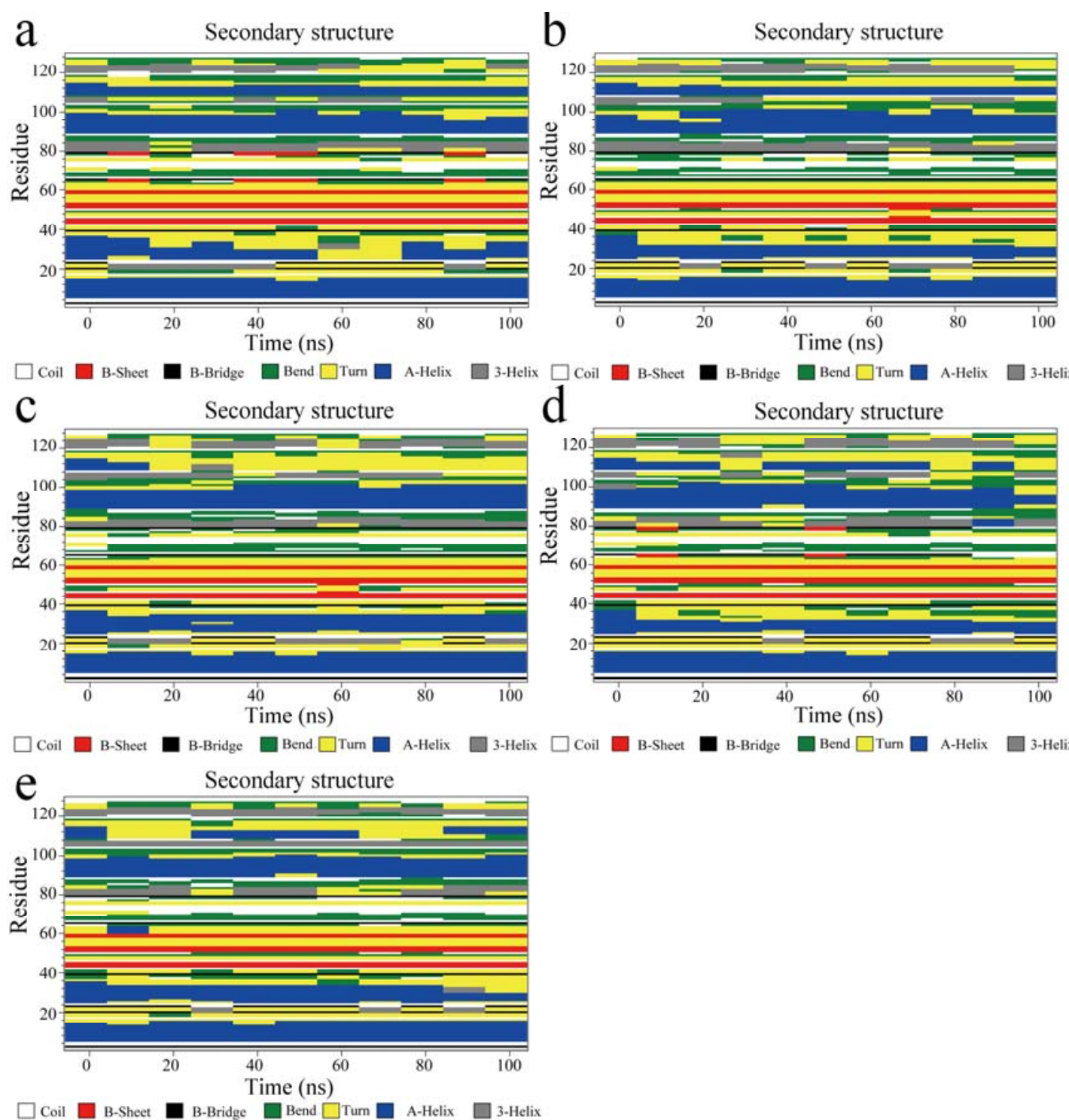

Figure S3. The secondary structure profile of lysozyme (a) in water and adsorption on the surface of CC3 in the (b) O1, (c) O2, (d) $\mathrm{O} 3$ and (e) $\mathrm{O} 6$ orientations. 

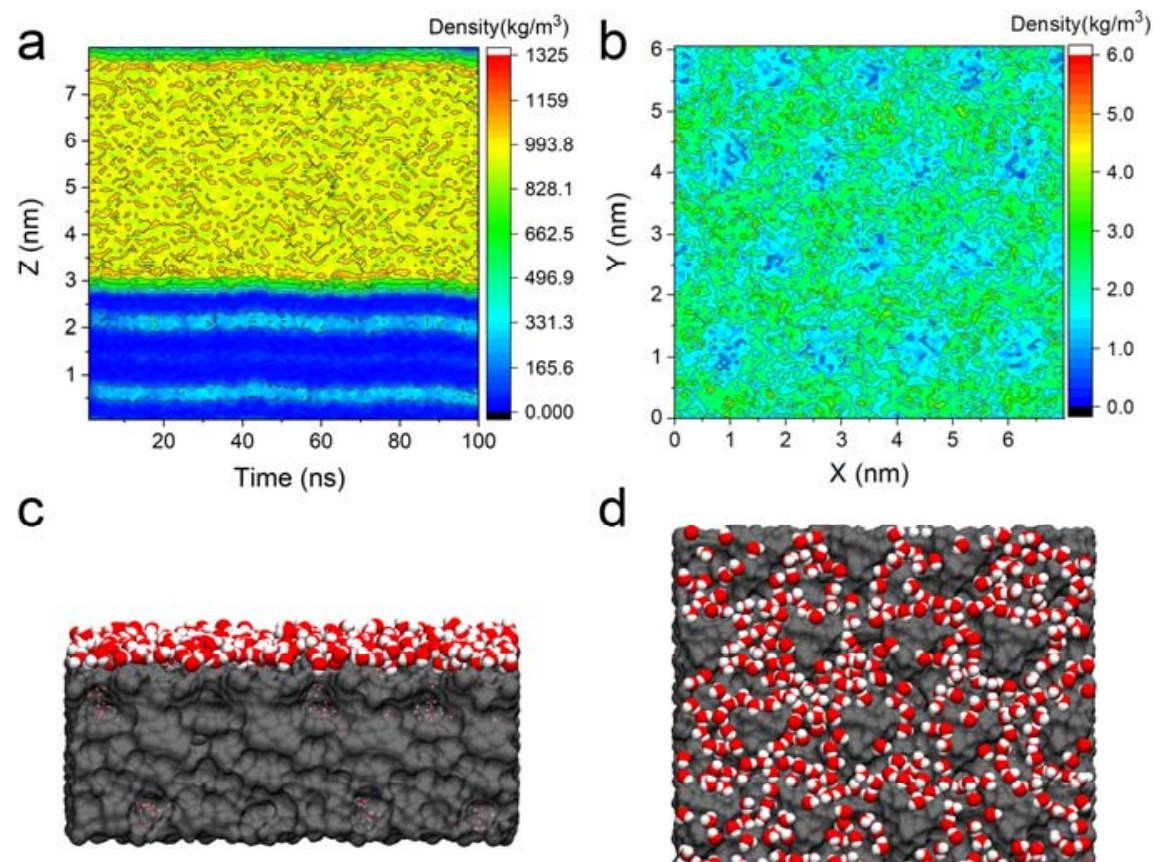

d

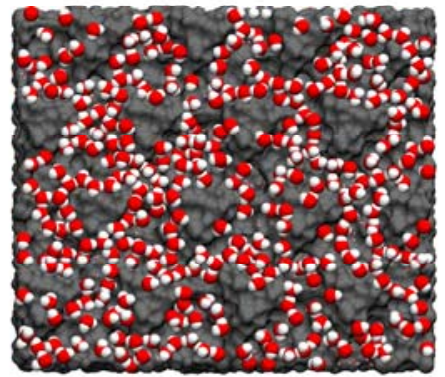

Figure S4. The density and configuration of water molecules on CC3 crystal without lysozyme. (a) Mass density distribution of water molecules along the z-axis. (b) Density map of the water layer above the CC3 surface at $z=2.8-3.1 \mathrm{~nm}$. (c) Side and (d) top view of the water-CC3 configuration. Only water molecules that reside at $z=$ 2.8-3.1 $\mathrm{nm}$ in the z-direction are shown in vdW spheres. The CC3 crystal is represented by surface.

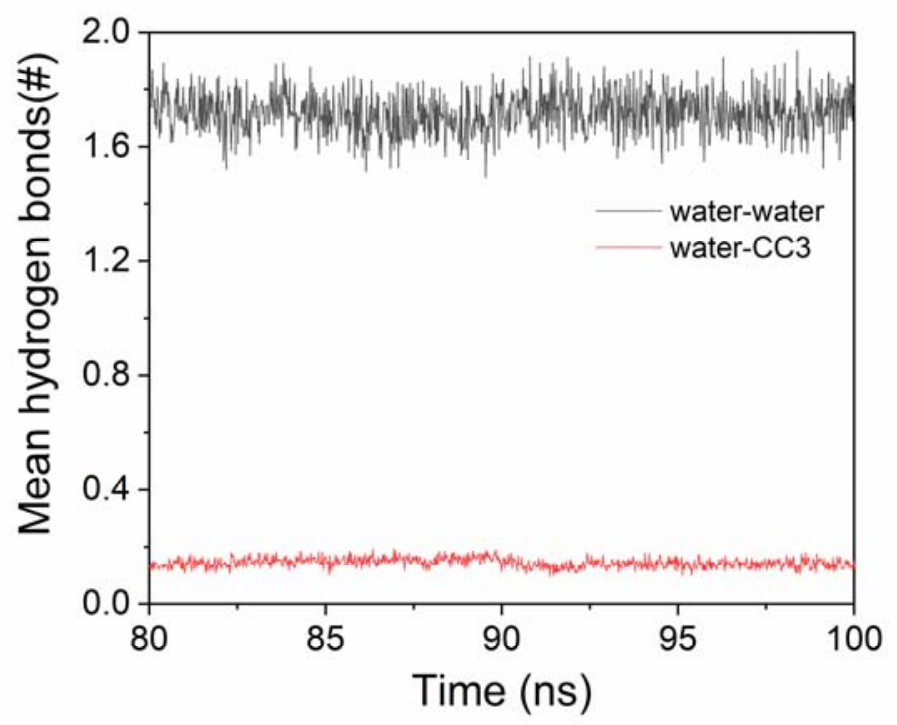

Figure S5. The average number of hydrogen bonds for interface water-water and interface water-CC3 at the last 20 ns MD simulation versus time. 